\title{
Coating Stresses in Thermal Barrier Coatings by an In-Situ Curvature Monitoring Technique
}

\author{
Masayuki ARAI** \\ **Materials Science Research Lab., Central Research Institute of Electric Power Industry, \\ 2-11-1 Iwado-kita, Komae-shi, Tokyo 201-8511, Japan \\ E-mail: marai@criepi.denken.or.jp
}

\begin{abstract}
In this paper, coating stresses in thermal barrier coating (TBC) changing with cyclic heating - cooling are measured with a curvature measurement device developed in this study. The coating system chosen in this study is a dual-layered structure, and it consists of a partially stabilized zirconia (PSZ) as the ceramic coating layer and CoNiCrAlY as the metal-bond coating layer. The specimen used here is a strip-plate shape with thin thickness $(600 \mu \mathrm{m})$ extracted chemically from carbon steel coated by a thermal spraying process. A cyclic heating-cooling test and a cyclic heating-cooling test with a dwelling time at the maximum temperature are conducted for the strip-plate specimen. Deflection and coating stresses are measured continuously under these cyclic tests, and thermal deformation mechanisms generating the deflection and coating stresses are discussed based on primitive knowledge using an elementary beam theory.
\end{abstract}

Key words: Ceramic Thermal Barrier Coating, Coating Stress, Thermal Cycling, Curvature Monitoring

\section{Introduction}

Cracking and peeling in thermal barrier coating (TBC), which is usually applied to the surface of important components in land-based gas turbines for preventing a high-temperature environment, are caused by thermal stress, i.e. coating stress, in addition to residual stress generated by the thermal spraying process. Therefore, it is quite important to clarify how the coating stress changes with temperature variation.

The stress-measurement method of monitoring the shift of selected X-ray diffraction peaks is well known and used widely to measure residual stress at a coating surface [1]. However, the results obtained by this X-ray diffraction method include a large amount of scattering for thermal sprayed coating especially. The main reason this large amount of scattering occurs is due to the fact that the penetration depth of X-rays is usually no greater than the surface roughness of sprayed coating. Additionally, it is also unsuitable to measure continuously coating stress that changes with cyclic temperature variation.

Another method is the stress-measurement method of monitoring the curvature of the coated specimen. This is a method of obtaining an average residual stress over a coating layer from the curvature of the coated specimen, which is caused by a mismatch of thermal expansions between two layers. The pioneer in this method is Stoney [2]. He applied this method to measure residual stress generated in a physical vapor deposited coating. In application to a thermal spraying process, Kuroda et al. [3][4] and Gill et al. [5][6] made clear the formation process of residual stress changed during the thermal spraying process by using this curvature monitoring technique. They developed individually a monitoring

*Received 9 May, 2008 (No. 08-0359) [DOI: 10.1299/jmmp.2.1207] 
device to measure the curvature inside the thermal spraying chamber. Kuroda applied a contact-type displacement sensor to measure the curvature of the specimen. For the same purpose, Gill specially developed a measurement system for monitoring the curvature from an image analysis performed based on video recordings. The former method might involve error caused by an external force occurring due to the specimen surface being pushed by the tip of the sensor. The latter has limitations for sampling time and flame resolution. Consequently, the monitoring device they developed still has some problems in precisely measuring curvature.

Now, it is known that coating stress changed with thermal cycling has quite interesting behavior, because the coating layer itself involves a lot of small grains and an unstable microstructure in much thinner than bulk materials. Koike et al. [7] performed some thermal cycling tests for silicon substrate specimen deposited aluminum alloy film by an RF magnetron-sputtering device and clarified that coating stress versus temperature curve obtained by thermal cycling has a hysteresis loop. It was found that coating stress remaining after thermal cycling increases with a cyclic number due to an increase in lattice dislocation and tangling. Gall et al. [8] tested Au-deposited polysilicon under almost the same cycling tests and examined creep deformation behavior in the Au layer caused by the strain constraint of the polysilicon layer. They reported that creep deformation advanced with recovery of the microstructure and initiation of micro-cracking in the Au layer. Dislocation accumulation and cracking initiation are dominant in these deformation mechanisms especially for a physically deposited layer.

Returning our attention to thermal sprayed coatings, there are few reports related to coating stress changes with thermal cycling. As is well known, thermal spraying deposition is a mechanical process conducted by impinging melting particles onto a substrate. Then, the microstructure in the as-sprayed coating layer is unstable for an exposure temperature, because it already involves numerous defects such as pore and micro-cracks induced by quenching stress. By an aspect of this microstructure, coating stress generated in thermally sprayed coating will change with strange behavior.

In this study, coating stress in TBC coating changed with thermal cycling was measured using a curvature measurement device developed herein. In this device, a long-range-type laser was used as a non-contacting sensor. The coating system chosen for this study is a dual-layered structure, and it consists of partially stabilized zirconia as the ceramic coating layer and CoNiCrAlY as the metal-bond coating layer. The specimen used here is a strip-plate shape with thin thickness $(600 \mu \mathrm{m})$, extracted chemically from the coated carbon steel. A cyclic heating-cooling test and a cyclic heating-cooling test with a dwelling time at the maximum temperature were conducted. Thermal deformation mechanisms generating the coating stress are also discussed based on primitive knowledge by an elementary beam theory.

\section{Experimental Procedure}

\subsection{TBC Sample Preparation}

TBC coated carbon steel was prepared for this study. The combination of the coating system used here is $8 \mathrm{wt} \% \mathrm{Y}_{2} \mathrm{O}_{3}-\mathrm{ZrO}_{2} / \mathrm{CoNiCrAlY}$. An $8 \mathrm{wt} \% \mathrm{Y}_{2} \mathrm{O}_{3}-\mathrm{ZrO}_{2}$ layer with thickness $0.5 \mathrm{~mm}$ and a CoNiCrAlY metal-bond layer with thickness $0.1 \mathrm{~mm}$ were coated by an atmospheric plasma spray (APS) technique on a plate-shaped carbon-steel substrate with geometry ${ }^{t} 1 \mathrm{X} 100 \mathrm{X} 100 \mathrm{~mm}$. After the APS process, a strip-shape coated sample with in-plane geometry $4 \mathrm{X} 40 \mathrm{~mm}$ was cut from the plate by a diamond cutter. Furthermore, an $8 \mathrm{wt} \% \mathrm{Y}_{2} \mathrm{O}_{3}-\mathrm{ZrO}_{2} / \mathrm{CoNiCrAlY}$ dual-layered sample was extracted with chemical treatment (a mixture of nitric acid $100 \mathrm{ml}$ and hydrochloric acid $300 \mathrm{ml}$ ) from the strip-shape coated sample. An initial center deflection $\delta_{0}$ of the dual-layered sample measured after extracting 
from the coated sample (that is, after being released from a strain constraint of the substrate) was $0.6 \mathrm{~mm}$. The aspect of the dual-layered sample used in following experiments is shown in Fig. 1.

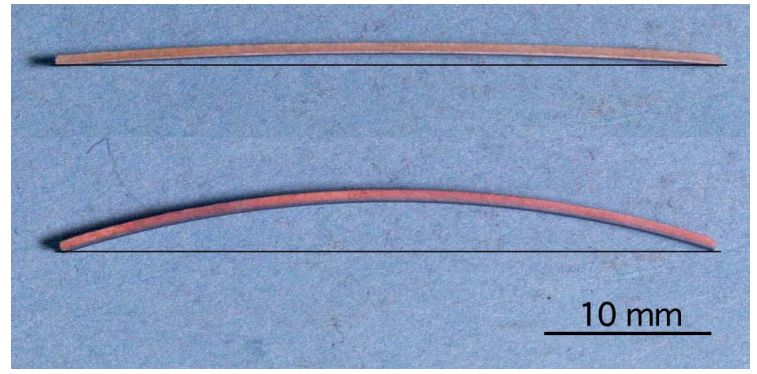

Fig. 1. Aspect of dual-layered sample used in this study; the upper photo shows the sample before the thermal cycling test, and the lower photo shows the sample shape after several cycles in the cyclic heating-cooling test.

\subsection{Curvature-monitoring System and Test Conditions}

An in-situ curvature-monitoring device used in this study is shown in Fig. 2. The dual-layered sample was put on a panel-type ceramic-heater. The deflection $\delta$ at the center position of the sample was measured by using a long-distance focused-type laser displacement sensor (Keyence LK-500). Temperature change during thermal cycling was also monitored by a CA-type thermocouple that was set near the sample, and also a ceramic-panel heater was controlled with a heater controller. Digital signals from the laser sensor and thermocouple were recorded on a personal computer. Here, the deflection indicated in the figure was taken as being in a positive direction.

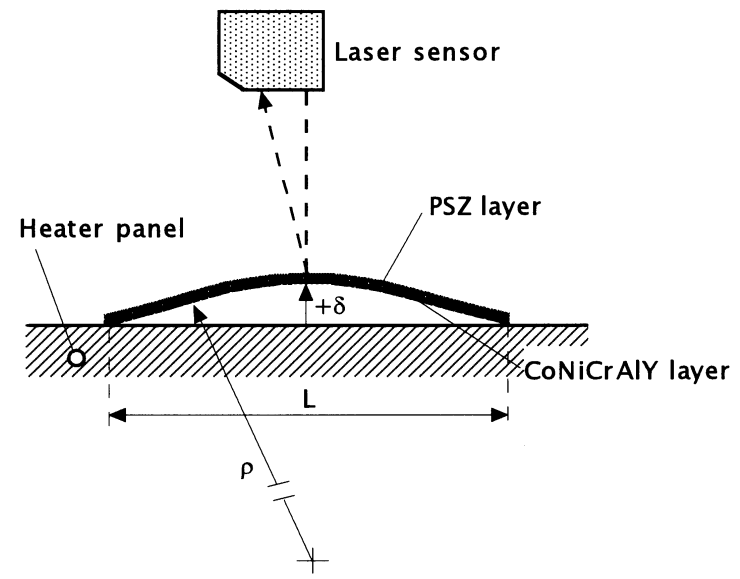

Fig. 2. Schematic diagram illustrating experimental arrangement.

In this study, the following three kinds of tests were conducted: (1) cycle test due to heating and cooling, (2) cyclic heating-cooling test with gradually increasing maximum temperature by each cycle and (3) cyclic heating-cooling test with dwelling time $100 \mathrm{~h}$ at the maximum temperature.

Through these tests, deflection and coating stress change with temperature were examined. 


\subsection{Conversion From Deflection to Coating Stress}

It was assumed that coating stresses in each layer can be determined simply from deflection in the dual-layered sample by allowing for when the coating beam deforms elastically.

By this assumption, conversion from deflection to coating stresses was conducted based on bi-metal analysis given by Timoshenko's model [9], [10], [11].

The dual-layered beam bonded completely together is assumed to be of uniform thickness $\left(h_{c}\right.$ in the ceramic coating layer and $h_{b}$ in the metal-bond coating layer) and with elastic modulus ( $E_{c}$ in the ceramic coating layer and $E_{b}$ in the metal-bond coating layer). The beam has length $\mathrm{L}$, and the width is assumed to be equal to unity. In this dual-layered beam subjected to any uniform temperature change from the reference temperature, each beam expands or shrinks according to a different thermal deformation, caused by the coefficient of thermal expansion. Axial forces $\left(\mathrm{P}_{c}\right.$ and $\left.\mathrm{P}_{\mathrm{b}}\right)$ and bending moments $\left(\mathrm{M}_{\mathrm{c}}\right.$ and $\mathrm{M}_{\mathrm{b}}$ ) over each section in these beams have to be generated to satisfy compatibility of displacement between the ceramic-coating and metal-bond-coating beams. Due to the fact that there is no external force at the edge of the dual-layered beam, the resultant force becomes zero:

$$
P_{c}+P_{b}=0
$$

For a resultant moment, this becomes:

$$
M_{c}+M_{b}-\frac{h_{c}+h_{b}}{2} P_{b}=0
$$

Letting $\rho$ the curvature radius of the dual-layered beam, the moments in each beam are:

$$
M_{c}=\frac{E_{c} h_{c}^{3}}{12 \rho}, M_{b}=\frac{E_{b} h_{b}^{3}}{12 \rho}
$$

Assuming that the deflection $\delta$ is small in comparison with the curvature radius $\rho$, we can have the following relation:

$$
\delta=\frac{L^{2}}{8 \rho}
$$

Using the relations from Eq. (1) to Eq. (4), coating stresses in each layer are given by:

$$
\begin{aligned}
\sigma_{c} & =-\frac{4}{3} \frac{E_{c} h_{c}^{3}+E_{b} h_{b}^{3}}{\left(h_{c}+h_{b}\right) h_{c} L^{2}} \delta \\
\sigma_{b} & =+\frac{4}{3} \frac{E_{c} h_{c}^{3}+E_{b} h_{b}^{3}}{\left(h_{c}+h_{b}\right) h_{b} L^{2}} \delta
\end{aligned}
$$

Under the assumption of plane strain, the term $E$ in Eq. (5) changes to $E /\left(1-v^{2}\right)$. However, the Poisson's ratio of freestanding ceramic coatings is a very small value such as 0.07 that was measured in advance by a cross-arranged strain gage attached on the coating surface. Thus, Eq. (5) is still available for this assumption in plane-strain conditions.

If the thickness of the ceramic coating layer $h_{c}$ is thinner than that of the metal-bond coating layer $h_{b}$, the coating stress in the ceramic coating layer in Eq. (5) reduces to: 


$$
\sigma_{c}=-\frac{4}{3} \frac{E_{b} h_{b}^{2}}{h_{c} L^{2}} \delta
$$

This equation is identified as Stoney's equation [2], as is well-known.

In conversion from deflection data measured experimentally to coating stresses, the following functions of elastic modulus with variable $\mathrm{T}$ as temperature, which was determined by a best-fitting for data obtained from a resonance measurement [12] using a free-standing layer, were used.

$$
\begin{aligned}
& E_{c}(T)=40.496-0.0014402 T-1.4235 \times 10^{-5} T^{2}+1.7717 \times 10^{-8} T^{3} \\
& E_{b}(T)=168.93-0.0618720 T+5.8513 \times 10^{-5} T^{2}-4.9644 \times 10^{-8} T^{3}
\end{aligned}
$$

where elastic modulus is unit in [GPa] and temperature is also unit in absolute temperature $[\mathrm{K}]$.

\section{Results and Discussion}

\subsection{Cyclic Heating-cooling test}

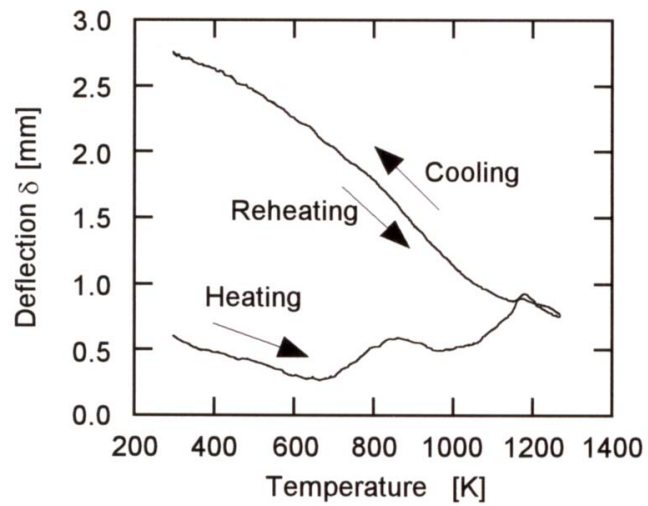

Fig. 3. Deflection versus temperature response including the initial deflection caused by release from the substrate

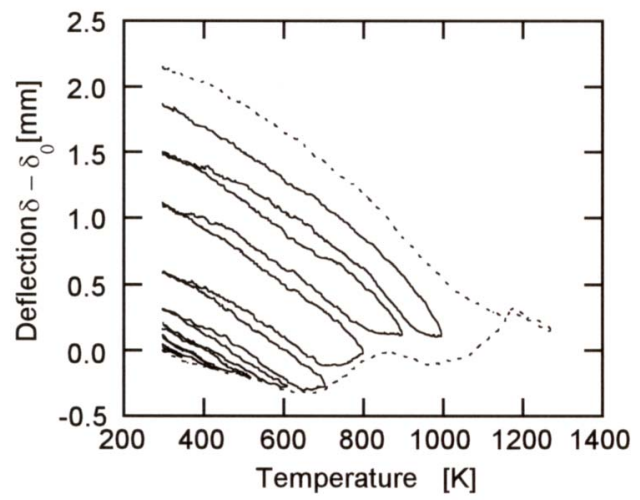

Fig. 4. Deflection versus temperature obtained by progressively increasing maximum temperature by each cycle 
Experimental data for the cyclic heating-cooling test is shown in Fig. 3. After release from the carbon-steel substrate at room temperature, the dual-layered beam had initial deflection of $\delta_{0}=0.6 \mathrm{~mm}$, as already mentioned in the section on TBC specimen preparation. At the start of the heating process, the deflection in the beam decreases with temperature. This decrease in deflection follows linear thermo-elastic behavior until the critical temperature of $700 \mathrm{~K}$ is reached. At this critical temperature, the relationship between the deflection in the dual-layered beam and temperature changes to a nonlinear one with unstable behavior, as shown in the figure. The deflection goes up for a little until the maximum temperature of $1273 \mathrm{~K}$ is reached. Starting cooling at the maximum temperature, the deflection increases almost linearly again. In the cyclic heating-cooling process conducted successively after this first cycle, deflection varied to the reverse of temperature along the line depicted during cooling in the first cycle. Experimental results obtained by progressively increasing maximum temperature values in each cycle are shown in Fig. 4. Maximum temperature was increased by $100 \mathrm{~K}$ with each cycle, up to $1273 \mathrm{~K}$. The vertical axis in this figure indicates a deflection difference from the initial deflection. The deflection in the heating process changes following the line depicted in the cooling process in the previous cycle. However, the deflection change has the limitation shown by the dotted line, which corresponds to results observed in the heating process in the monotonic cyclic heating-cooling test. In the cooling process after reaching the maximum temperature, deflection increased linearly again.

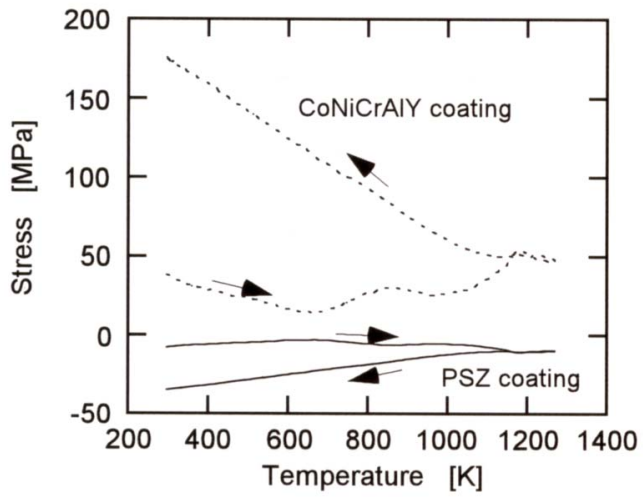

Fig. 5. Coating stress versus temperature obtained from cyclic heating-cooling test

Results for coating stresses converted from Fig. 3 are shown in Fig. 5. Residual stress in each coating layer calculated from the initial deflection was $-17 \mathrm{MPa}$ in the ceramic coating layer and $49 \mathrm{MPa}$ in the metal-bond coating layer. During the heating-cooling process, the coating stress in the ceramic coating layer changed over a negative range, i.e. over the compressive stress state. Coating stress in metal-bond coating layer changed over a tensile stress state in contrast with the stress in ceramic coating layer, and its maximum value became about $180 \mathrm{MPa}$.

Here, we have some questions from these cyclic heating-cooling test results, as following.

(1) What does critical temperature as a transient point from linear to nonlinear behavior mean, physically?

(2) How does each beam in the dual-layered beam structure deform thermally during the cyclic heating-cooling process, and why does the deflection of the dual-layered beam cause strange behavior as shown in Fig. 3?

Firstly, we attend to the apparent process temperature $\mathrm{T}_{\mathrm{p}}$, which is defined as average temperature over coatings, distributed when the coating layer is exposed during the thermal 
spraying process. For this, we tried to estimate the process temperature by an extra experiment as follows: a thermocouple was spot welded onto the rear side of the substrate, and the substrate temperature was monitored continuously during the APS process. Then, the process temperature was estimated by performing thermal conduction analysis based on the monitored substrate temperature. These analysis results made clear that the process temperature $T_{p}$ in the ceramic coating layer should be equal to $736 \mathrm{~K}$ in our process condition in APS. Remembering here that the critical temperature observed in Fig. 3 was about $700 \mathrm{~K}$, it can be concluded that critical temperature means a process temperature $T_{p}$ over a coating layer exposed during the thermal spraying process. Thus, because the as-sprayed coating has already experienced a high temperature up to the process temperature $T_{p}$, the microstructure in the ceramic coating maintains stability until the test temperature reaches the process temperature. Through this stability, deflection generated in the dual-layered beam decreased linearly with temperature until reaching the process temperature $T_{p}$. The nonlinear deflection behavior appeared at a higher temperature than the process temperature $T_{p}$ because of an unstable and inexperienced microstructure for temperature.

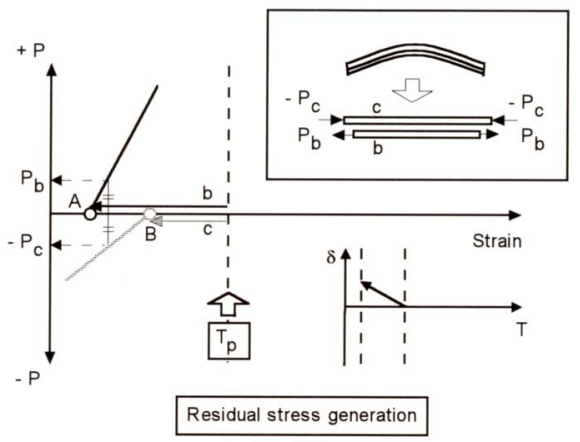

Fig. 6. Illustration describing the deformation process after release from the substrate
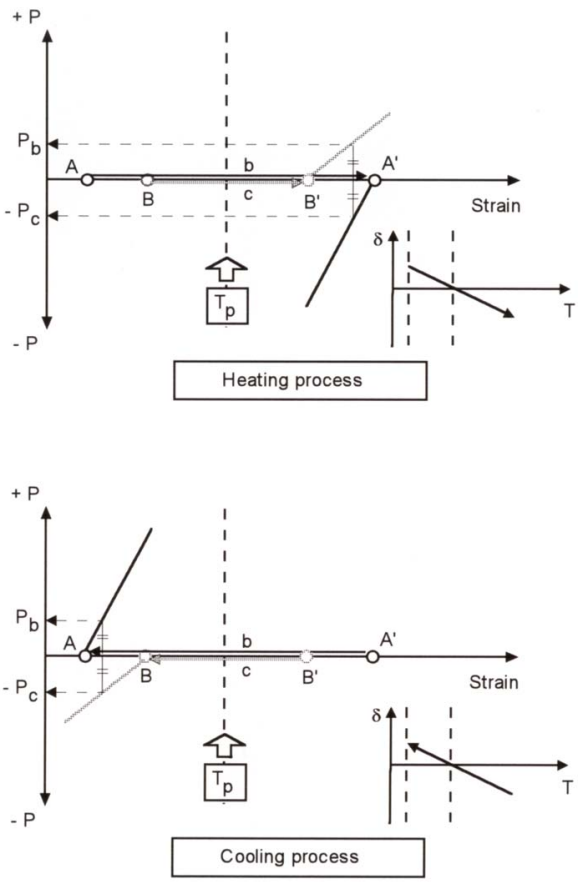

Fig. 7. Illustration for explaining thermal deformation during heating and cooling process conducted as a thinking test. 
Successively, we will discuss thermal deformation generating deflection in the dual-layered beam. A schematic illustration for considering its mechanisms is shown in Fig. 6. The vertical axis shows the axial force enforced in each beam and the horizontal axis shows strain including that of both mechanical and thermal components. At process temperature $T_{p}$, the beam length of the ceramic coating layer should be equal to the length of the bond coating layer. After finishing the thermal spraying process, both the ceramic coating and metal-bond coating beams shrink simultaneously, with thermal strain generated until reaching room temperature (i.e., the length of the ceramic coating beam shrinks along line $\mathrm{c}$ and the length of the metal-bond coating shrinks along line $\mathrm{b}$; thus, both lengths reach points $\mathrm{A}$ and $\mathrm{B}$ at room temperature). From compatibility of the deformation for beam shrinkage, axial tensile force $\mathrm{P}_{\mathrm{b}}$ in metal-bond coating beam and compressive force $\mathrm{P}_{\mathrm{c}}$ in ceramic coating beam, which is equivalent to absolute value of $\mathrm{P}_{\mathrm{b}}$, have to be enforced as shown in the figure. Mechanical strain caused by these forces brings about deflection of the dual-layered beam and residual stresses, which were observed as initial deflection $\delta_{0}$ after release from the substrate.
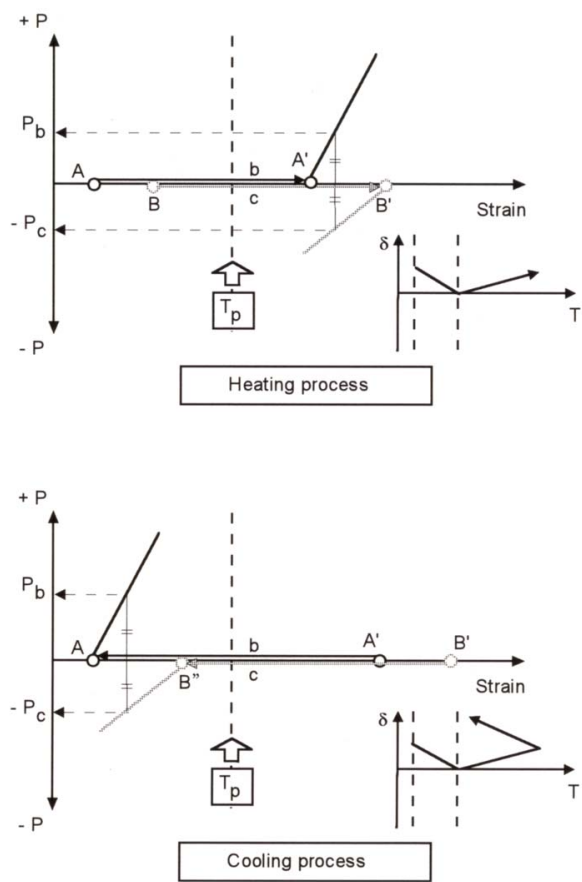

Fig. 8. Illustration for explaining experimental results consistently

Next, if we conduct a heating test from this initial state (i.e., points A and B in Fig. 7) to the maximum temperature, the difference between the beam lengths at room temperature is canceled out when the test temperature has reached the process temperature. Further heating process would bring an inversion of beam lengths between the ceramic-coating and bond-coating layers (i.e., point A' and B' in Fig. 7). This inversion of beam lengths causes a negative deflection in the dual-layered beam (i.e., a concave shape of the beam). Starting cooling again at maximum temperature, the deflection increases linearly along the deflection line depicted in a heating process and should come back to the initial deflection value when reaching room temperature. However, it should be noted that the result obtained by this thinking test is quite different from the actual result as observed in Fig. 3! For a consistent explanation to the experiment, one must employ extraneously the fact that the length of the ceramic coating beam is still larger than the length of the metal-bond coating beam in higher temperatures than the process temperature $T_{p}$. A schematic illustration for explaining this situation is shown in Fig. 8. This illustration demonstrates that point $\mathrm{A}$ as the 
initial beam length of metal-bond coating goes to point A' with thermal expansion. Point B as the initial state of ceramic coating goes to point B' across point A'. Revealing this consistent explanation with more physical meaning, including microstructure changes, is a topic for future research.

\subsection{Cyclic heating-cooling Test with Dwelling Time}

The experimental results for a cyclic heating-cooling test with dwell time $100 \mathrm{~h}$ at maximum temperature is shown in Fig. 9. Results are included for maximum temperatures $673 \mathrm{~K}, 873 \mathrm{~K}$ and $1173 \mathrm{~K}$. The dotted line indicates that results varied through the heating process in all tests, and this line was almost the same as the heating results obtained in Fig. 3. Arrows correspond to deflection changes during dwelling times up to $100 \mathrm{~h}$. Also, solid lines are curves obtained by the cooling process after dwelling. Deflections during dwelling time at maximum temperature go up or down depending on the value of the maximum temperature. That is, dwelling at a higher temperature brings about a decrease of deflection, and dwelling at a lower temperature brings about an increase of deflection. Fig. 10 shows variation of deflection with dwelling time. This result indicates that deflections change gradually with time, and deflection in a lower maximum temperature (673 and $873[\mathrm{~K}]$ ) increases with dwelling time and in a higher maximum temperature (1073 and 1273[K]) decreases with time, in quite a contrast with the lower results.

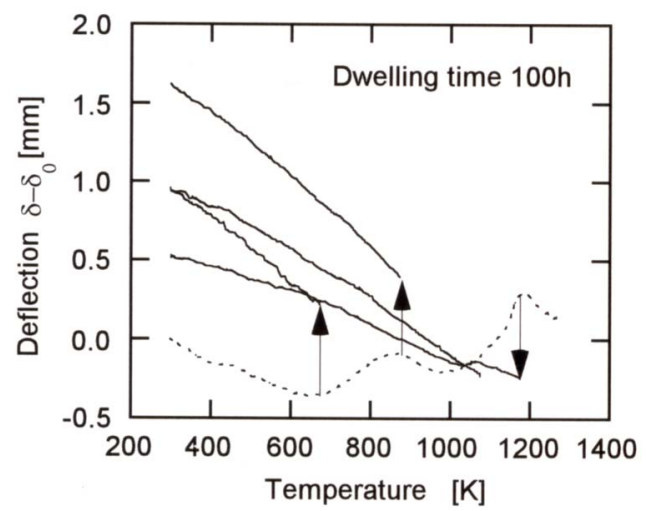

Fig. 9. Deflection versus temperature response in cyclic heating-cooling test with dwell time at a maximum temperature.

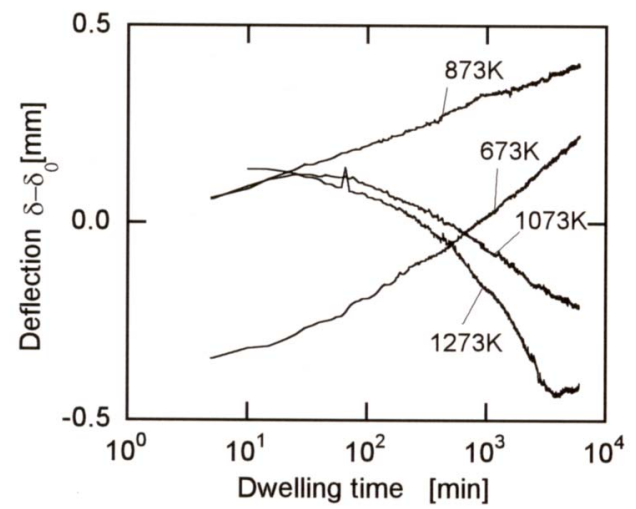

Fig. 10. Deflection varying with time while maintaining maximum temperature. Deflection indicates difference from the initial deflection. 
Fig. 11 shows results for coating stresses converted from the deflection data versus dwelling time. Coating stress in the ceramic coating is in a negative range, i.e. in compressive stress state, over dwelling time independently of the condition of the maximum temperature. On the other hand, coating stress in metal-bond coating is tensile over the dwelling time. Coating stresses in both CoNiCrAlY and PSZ approaches to zero level with dwelling time in maximum temperature 1073 and $1273[\mathrm{~K}]$.

Generally speaking, deflection is considered to decrease with dwelling time independently of test conditions of the maximum temperature, because the metal-bond coating has low yield stress and low resistance for creep deformation and gradually elongates with dwelling time by being subjected to tensile force $\mathrm{P}_{\mathrm{b}}$. However, our experimental results indicated inverse behavior to this. This is another case of strange behavior!

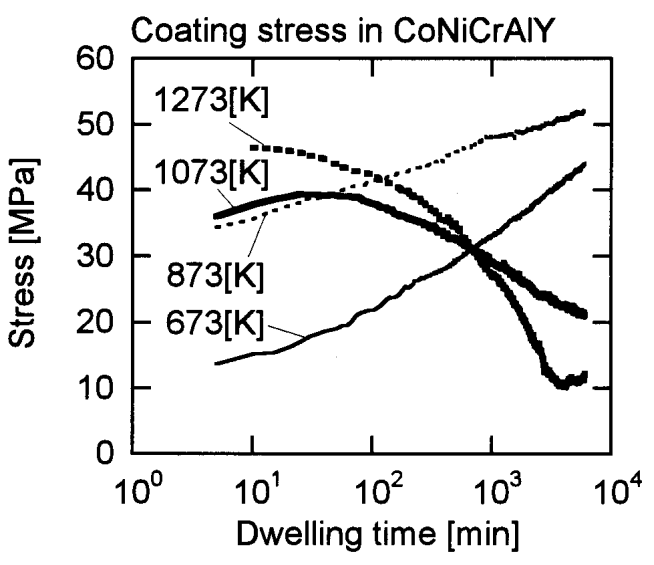

(a) Coating stress in CoNiCrAlY

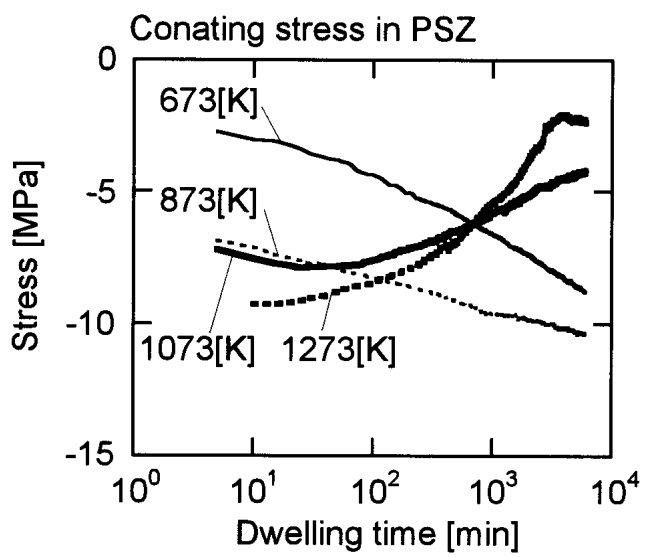

(b) Coating stress in PSZ

Fig. 11 Coating stresses varying while maintaining maximum temperature

The microstructure of a typical as-sprayed TBC coating is shown in Fig. 12. The ceramic coating layer involves a lot of globular pores, intrasplat and micro-cracks. Here, we examined how the microstructure of as-sprayed TBC changes after 100 cycles in cyclic heating-cooling tests with dwelling time. Quantification of porosity and average diameter of pores was carried out for this purpose. The result is shown in Fig. 13. Porosity decreases slowly at a lower maximum temperature than process temperature $T_{p}$, and the average diameter of pores increases rapidly, in contrast with the results for porosity. At a higher 
maximum temperature than $T_{p}$, both porosity and the average diameter of pores decrease at almost the same rate. Decreased porosity means to be a strong bind between spray particles in the coating layer, and an increased average diameter of pores means swelling of the apparent volume of ceramic coating, basically. Thus, it appeared as a time-dependent deformation in ceramic coating that the swelling deformation of the ceramic coating layer occurred while dwelling temperature was lower than process temperature $T_{p}$, and by this deformation mechanism deflection increased with dwelling time. But having careful notice for result of $873[\mathrm{~K}]$, it was observed that the associated deflection increases with dwelling time, which we can not completely give explanation to this. At a higher temperature in dwelling, deflection decreased with dwelling time due to creep deformation in the metal-bond coating layer. Here, it should be noticed that thermally grown oxidation (TGO) is formed at interface between ceramic and bond coating under high-temperature exposure. TGO formation changes layered structure from dual to triple. However, it is known [13] that TGO is formed at exposure temperature higher than $1073[\mathrm{~K}]$. Thus, in cyclic test case of $673[\mathrm{~K}]$ and $873[\mathrm{~K}]$, coating stress generation mechanism associated with holding period at maximum temperature is still supported by swelling deformation of the ceramic coating. In test case of $1073[\mathrm{~K}]$ and $1273[\mathrm{~K}]$, creep deformation would be dominant during holding period, but amount of creep deformation could be affected by TGO formation.

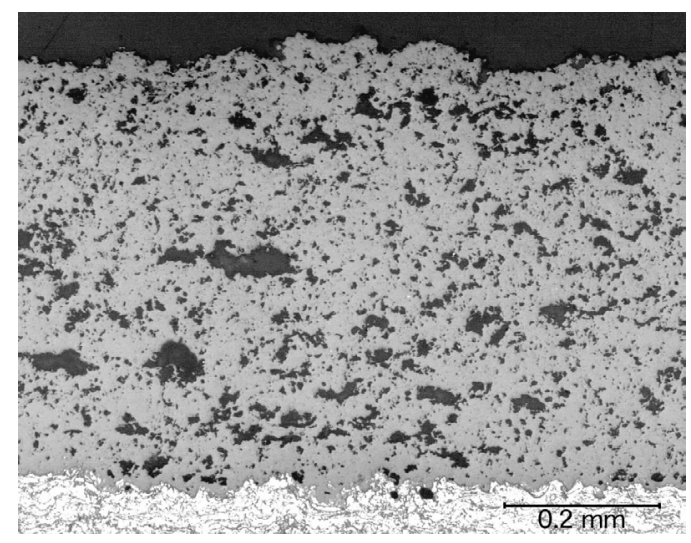

Fig. 12. Microstructure of as-sprayed TBC coating

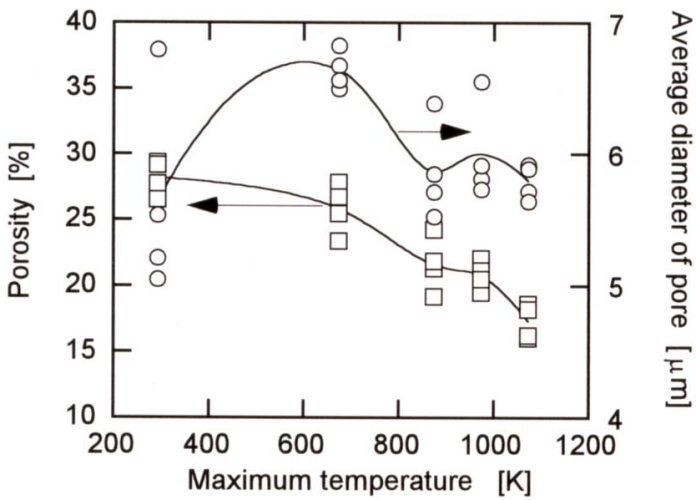

Fig. 13. Porosity and average diameter of pore in TBC coating after subjection to 100 -cycle heating-cooling test with dwelling time at maximum temperature, which was set up as a test condition 


\section{Conclusion}

In this paper, deflection and coating stresses converted from deflection data in thermal barrier coating (TBC) varying with a cyclic heating-cooling process were measured by a curvature monitoring technique. The coating system chosen in this study was a dual-layered structure, consisting of a partially stabilized zirconia and CoNiCrAlY. The specimen also was a thin strip-plate shape with thickness $600 \mu \mathrm{m}$. A cyclic heating-cooling test and a cyclic cooling test with dwelling time at maximum temperature were conducted. In results obtained through an in-situ curvature monitoring experiment, it was observed in the cyclic heating-cooling test that the deflection decreased linearly until reaching the process temperature and increased a little up to the maximum temperature. Coating stress in the ceramic coating layer was compressive over the thermal cycle, and coating stress in the metal-bond coating layer was tensile. This deflection versus temperature response was in opposition to results of simulation based on elementary beam theory, and it is quite an interesting phenomenon. In a cyclic heating-cooling test with dwelling time at maximum temperature, deflection increased with dwelling time at a lower maximum temperature than the process temperature. On the other hand, the deflection decreased with dwelling time at a higher maximum temperature. The latter phenomenon could be explained easily by a creep deformation mechanism based on the strain constraint of the ceramic coating layer. However, the former could not be explained by the same mechanism. Herein, a swelling mechanism in the ceramic coating layer as a time-dependent deformation was considered for overcoming this difficulty.

We need to consider more deeply what microscopic deformation mechanisms dependent on test temperature are present in ceramic coating sprayed thermally.

\section{References}

(1) M. Murakami, Residual stress of $\mathrm{Pb}$ thin films deposited onto Si substrates, Acta metall, Vol.26 (1978), pp.175-183.

(2) G. G. Stoney, The tension of metallic films deposited by electrolysis, Proc roy soc A, Vol. 32 (1908), pp.172-175.

(3) S. Kuroda, T. Fukushima, S. Kitahara, Simultaniuous measurement of coating thickness and deposited stress during thermal spray, Thin solid films, Vol.164 (1988), pp.157-163.

(4) S. Kuroda, T. W. Clyne, The quenching stress in thermally sprayed coatings, Thin solid films, Vol.200 (1991), pp.49-66.

(5) S. C. Gill, T. W. Clyne, Stress distributions and material response in thermal spraying of metallic and ceramic deposits, Metall trans B, Vol. 21 (1990), pp.377-385.

(6) S. C. Gill, T. W. Clyne, Investigation of residual stress generation during thermal spraying by continuous curvature measurement, Thin solid films, Vol. 250 (1994), pp.172-180.

(7) J. Koike, S. Utsunomiya, Y. Shimoyama, K. Murakami, H. Oikawa, Thermal cycling fatigue and deformation mechanism in aluminum alloy thin films on silicon", $J$ mater res, Vol. 13, No.11 (1998), pp.3256-3264.

(8) K. Gall, N. West, K. Spark, M. L. Dunn, D. S. Finch, Creep of thin film Au on bimaterial $\mathrm{Au} / \mathrm{Si}$ microcantilevers, Acta met, Vol. 52 (2004), pp.233-255.

(9) S. Timoshenko, Analysis in bi-metal thermostats, J.O.S.A. \& R. S. I., Vol. 11 (1925), pp.233-255.

(10) E. Suhir, Stress in bi-metal thermostats, ASME J of appl mech, Vol. 53 (1986), pp.657-660.

(11) P. H. Townsend, D. M. Barnett, T. A. Brunner, Elastic relationships in layered composite media with approximation for the case of thin films on a thick substrate, $J$ appl phys, Vol. 62, No. 11 (1987), pp.4438-4444.

(12) M. Arai, K. Kishimoto, Estimation method of Young's modulus of thermal barrier coating 
layer based on free bending vibration, J of soc mat sci JPN, Vol. 52, No. 9 (2003), pp.1135-1139.

(13) M. Arai, U. Iwata, H. Satoh and K. Kishimoto, On Interface Oxidation Process of $8 \mathrm{wt} \% \mathrm{Y}_{2} \mathrm{O}_{3}-\mathrm{ZrO}_{2} / \mathrm{CoNiCrAlY}$ Thermal Barrier Coating, Trans. of JPN soc. of mech. eng., Vol. 69, No.678 (2003), pp.245-250. 